\title{
Existence of positive solutions for a boundary value problem of nonlinear fractional differential equations
}

\author{
Yude $\mathrm{Ji}^{1 *}$, Yanping Guo ${ }^{2}$, Jiqing Qiu and Liyun Yang ${ }^{1}$
}

\section{"Correspondence:}

jiyude-1980@163.com

'College of Sciences, Hebei

University of Science and

Technology, Shijiazhuang, Hebei 050018, P.R. China

Full list of author information is

available at the end of the article

\begin{abstract}
In this paper, we investigate the existence and multiplicity results of positive solutions for a boundary value problem of nonlinear fractional differential equations. The differential operator is taken in the Riemann-Liouville sense and the nonlinear term depends on the fractional derivative of an unknown function. We derive first the associated Green's function and prove its properties. Consequently, the considered problem is deduced to an equivalent integral equation. Next, by means of the Leggett-Williams fixed point theory, we obtain the existence and multiplicity results of positive solutions.
\end{abstract}

Keywords: boundary value problem; fractional differential equation; fixed point theorem

\section{Introduction}

Fractional derivatives are generalizations for derivative of integral order. There are several kinds of fractional derivatives, such as Riemann-Liouville fractional derivative, Marchaud fractional derivative, Caputo's derivative, Griinwald-Letnikov fractional derivative, etc. In the last few decades, fractional-order models have been found to be more adequate than integer-order models for some real world problems. Fractional derivatives provide an excellent tool for the description of memory and hereditary properties of various materials and processes. This is the main advantage of fractional differential equations in comparison with classical integer-order models. Fractional differential equations arise in many engineering and scientific disciplines as the mathematical modeling of systems and processes in the fields of physics, chemistry, aerodynamics, electrodynamics of complex medium, polymer rheology, and so forth, involves derivatives of fractional order. Fractional differential equations also serve as an excellent tool for the description of hereditary properties of various materials and processes. In consequence, the subject of fractional differential equations is gaining much importance and attention. For details, see [1-9] and the references therein. In [10-17], the authors discussed the existence of solutions for boundary value problems (BVPs) of nonlinear fractional differential equations. There is a large number of papers dealing with the solvability of nonlinear fractional differential equations. However, the theory of BVPs for nonlinear fractional differential equations is still in the initial stages and many aspects of this theory need to be explored. 
In [18], Bai and Lü used some fixed point theorems on cone to show the existence and multiplicity of positive solutions for a Dirichlet-type problem of the nonlinear fractional differential equation

$$
\left\{\begin{array}{l}
D_{0+}^{\alpha} u(t)+f(t, u(t))=0, \quad 0<t<1 \\
u(0)=u(1)=0
\end{array}\right.
$$

where $1<\alpha \leq 2$ is a real number, $D_{0+}^{\alpha}$ is a fractional derivative in the sense of RiemannLiouville, and $f:[0,1] \times[0,+\infty) \rightarrow[0,+\infty)$ is continuous.

In [19], Li et al. considered the following three point BVPs of fractional order differential equations

$$
\left\{\begin{array}{l}
D_{0^{+}}^{\alpha} u(t)+f(t, u(t))=0, \quad 0<t<1 \\
u(0)=0, \quad D_{0^{+}}^{\beta} u(1)=a D_{0^{+}}^{\beta} u(\xi)
\end{array}\right.
$$

where $D_{0^{+}}^{\alpha}$ is the Riemann-Liouville fractional derivative of order $1<\alpha \leq 2,0 \leq \beta \leq 1$, $0 \leq a \leq 1, \xi \in(0,1), a \xi^{\alpha-\beta-2} \leq 1-\beta, 0 \leq \alpha-\beta-1$ and $f:[0,1] \times[0,+\infty) \rightarrow[0,+\infty)$ satisfies Carathéodory type conditions.

Motivated by the result of $[18,19]$, we are concerned with the nonlinear differential equation of fractional order

$$
\left\{\begin{array}{l}
D_{0^{+}}^{\alpha} u(t)+f\left(t, u(t), D_{0^{+}}^{\mu} u(t)\right)=0, \quad 0<t<1, \\
u(0)=0, \quad u(1)+D_{0^{+}}^{\beta} u(1)=k u(\xi)+l D_{0^{+}}^{\beta} u(\eta),
\end{array}\right.
$$

where $D_{0^{+}}^{\alpha}$ is the Riemann-Liouville fractional derivative of order $1<\alpha \leq 2,0 \leq \beta \leq 1$, $\xi, \eta \in(0,1), 0 \leq \mu<1,1 \leq \alpha-\beta, 1 \leq \alpha-\mu, 1-k \xi^{\alpha-1} \geq 0,1-l \eta^{\alpha-\beta-1} \geq 0$, and $f:[0,1] \times$ $[0,+\infty) \times(-\infty,+\infty) \rightarrow[0,+\infty)$ is continuous.

To obtain positive solutions of BVP (1.1), the following fixed point theorem in cones, which can be found in [20], is fundamental.

Theorem 1.1 (Leggett-Williams fixed point theorem) Let $P$ be a cone in a real Banach space $E, P_{c}=\{x \in P \mid\|x\| \leq c\}, \theta$ be a nonnegative continuous concave functional on $P$ such that $\theta(x) \leq\|x\|$ for all $x \in \bar{P}_{c}$, and $P(\theta, b, d)=\{x \in P \mid b \leq \theta(x),\|x\| \leq d\}$. Suppose that $T: \bar{P}_{c} \rightarrow \bar{P}_{c}$ is completely continuous and there exist constants $0<a<b<d \leq c$ such that

$\left(C_{1}\right)\{x \in P(\theta, b, d) \mid \theta(x)>b\} \neq \emptyset$, and $\theta(T x)>b$ for $x \in P(\theta, b, d)$;

$\left(C_{2}\right)\|T x\|<a$ for $\|x\| \leq a$;

$\left(C_{3}\right) \theta(T x)>b$ for $x \in P(\theta, b, c)$, with $\|T x\|>d$.

Then $T$ has at least three fixed points $x_{1}, x_{2}$ and $x_{3}$, which satisfy

$$
\left\|x_{1}\right\|<a, \quad b<\theta\left(x_{2}\right) \text { and } a<\left\|x_{3}\right\| \quad \text { with } \theta\left(x_{3}\right)<b .
$$

Remark 1.2 If there holds $d=c$, then condition $\left(\mathrm{C}_{1}\right)$ implies condition $\left(\mathrm{C}_{3}\right)$ of Theorem 1.1.

The rest of this paper is organized as follows. In Section 2, we introduce some basic definitions, the associated Green's function and its properties are derived. In Section 3, we obtain the existence and multiplicity results of positive solutions for BVP (1.1). 
The following assumptions will stand throughout this paper:

$\left(\mathrm{H}_{1}\right) 1<\alpha \leq 2,0 \leq \beta \leq 1, \xi, \eta \in(0,1), 0 \leq \mu<1,1 \leq \alpha-\beta, 1 \leq \alpha-\mu, 1-k \xi^{\alpha-1} \geq 0$, $1-l \eta^{\alpha-\beta-1} \geq 0$

$\left(\mathrm{H}_{2}\right) f:[0,1] \times[0,+\infty) \times(-\infty,+\infty) \rightarrow[0,+\infty)$ is continuous.

\section{Basic definition and preliminary results}

In this section, we introduce preliminary facts and some basic results, which are used throughout this paper [21, 22].

Definition 2.1 The Riemann-Liouville fractional integral of order $\alpha>0$ for a function $y:(0, \infty) \rightarrow R$ is defined as

$$
I_{0^{+}}^{\alpha} y(t)=\frac{1}{\Gamma(\alpha)} \int_{0}^{t} \frac{y(s)}{(t-s)^{1-\alpha}} \mathrm{d} s, \quad \alpha>0
$$

provided the right-hand side is pointwise defined on $(0, \infty)$.

Definition 2.2 The Riemann-Liouville fractional derivative of order $\alpha>0$ for a function $y:(0, \infty) \rightarrow R$ is defined by

$$
D_{0^{+}}^{\alpha} y(t)=\frac{1}{\Gamma(n-\alpha)}\left(\frac{\mathrm{d}}{\mathrm{d} t}\right)^{n} \int_{0}^{t} \frac{y(s)}{(t-s)^{\alpha-n+1}} \mathrm{~d} s, \quad n=[\alpha]+1
$$

provided the right-hand side is pointwise defined on $(0, \infty)$.

Lemma 2.3 Let $\alpha>0$. If we assume $u \in C(0,1) \cap L(0,1)$, then the fractional differential equation $D_{0^{+}}^{\alpha} u(t)=0$ has the unique solution

$$
u(t)=c_{1} t^{\alpha-1}+c_{2} t^{\alpha-2}+\cdots+c_{n} t^{\alpha-n}
$$

where $c_{i} \in R, i=1,2, \ldots, n(n=[\alpha]+1)$.

Lemma 2.4 Assume that $u \in C(0,1) \cap L(0,1)$ with a fractional derivative of order $\alpha>0$ that belongs to $u \in C(0,1) \cap L(0,1)$. Then

$$
I_{0^{+}}^{\alpha} D_{0^{+}}^{\alpha} u(t)=u(t)+c_{1} t^{\alpha-1}+c_{2} t^{\alpha-2}+\cdots+c_{n} t^{\alpha-n}
$$

for some $c_{i} \in R, i=1,2, \ldots, n$.

Remark 2.5 [12] The following properties are useful for our discussion:

(i) $I_{0^{+}}^{\alpha} I_{0^{+}}^{\beta} y(t)=I_{0^{+}}^{\alpha+\beta} y(t), D_{0^{+}}^{\alpha} I_{0^{+}}^{\alpha} y(t)=y(t), \alpha>0, \beta>0, y(t) \in L(0,1)$;

(ii) $I_{0^{+}}^{\alpha} D_{0^{+}}^{\alpha} y(t)=y(t), 0<\alpha<1, y(t) \in C[0,1]$ and $D_{0^{+}}^{\alpha} y(t) \in C(0,1) \cap L(0,1)$;

(iii) $I_{0^{+}}^{\alpha}: C[0,1] \rightarrow C[0,1], \alpha>0$.

Lemma 2.6 [19] Assume that $y(t) \in L[0,1]$ and $\alpha, \beta$ are two constants such that $\alpha>1 \geq$ $\beta \geq 0$. Then

$$
D_{0^{+}}^{\beta} \int_{0}^{t}(t-s)^{\alpha-1} y(s) \mathrm{d} s=\frac{\Gamma(\alpha)}{\Gamma(\alpha-\beta)} \int_{0}^{t}(t-s)^{\alpha-\beta-1} y(s) \mathrm{d} s .
$$


In the following, we present Green's function of the fractional differential equation BVP.

Lemma 2.7 Given $y \in C[0,1]$ and $1<\alpha \leq 2$, the problem

$$
\left\{\begin{array}{l}
D_{0^{+}}^{\alpha} u(t)+y(t)=0, \quad 0<t<1 \\
u(0)=0, \quad u(1)=0
\end{array}\right.
$$

is equivalent to

$$
u(t)=\int_{0}^{1} G_{1}(\alpha, 0 ; t, s) y(s) \mathrm{d} s
$$

where

$$
G_{1}(\alpha, 0 ; t, s)=\frac{1}{\Gamma(\alpha)} \begin{cases}t^{\alpha-1}(1-s)^{\alpha-1}-(t-s)^{\alpha-1}, & 0 \leq s \leq t \leq 1 \\ t^{\alpha-1}(1-s)^{\alpha-1}, & 0 \leq t \leq s \leq 1 .\end{cases}
$$

Proof In view of Lemma 2.3, the equation $D_{0^{+}}^{\alpha} u(t)+y(t)=0$ is equivalent to the integral equation

$$
u(t)=-\frac{1}{\Gamma(\alpha)} \int_{0}^{t}(t-s)^{\alpha-1} y(s) \mathrm{d} s+c_{1} t^{\alpha-1}+c_{2} t^{\alpha-2}
$$

The boundary condition $u(0)=0$ implies that $c_{2}=0$. Thus

$$
u(t)=-\frac{1}{\Gamma(\alpha)} \int_{0}^{t}(t-s)^{\alpha-1} y(s) \mathrm{d} s+c_{1} t^{\alpha-1}
$$

In view of the boundary condition $u(1)=0$, we conclude that

$$
c_{1}=\frac{1}{\Gamma(\alpha)} \int_{0}^{1}(1-s)^{\alpha-1} y(s) \mathrm{d} s
$$

Therefore, the unique solution of problem (2.1) is

$$
u(t)=-\frac{1}{\Gamma(\alpha)} \int_{0}^{t}(t-s)^{\alpha-1} y(s) \mathrm{d} s+\frac{t^{\alpha-1}}{\Gamma(\alpha)} \int_{0}^{1}(1-s)^{\alpha-1} y(s) \mathrm{d} s .
$$

The unique solution of (2.1) is expressed as

$$
\begin{aligned}
u(t) & =\frac{1}{\Gamma(\alpha)} \int_{0}^{t}\left[t^{\alpha-1}(1-s)^{\alpha-1}-(t-s)^{\alpha-1}\right] y(s) \mathrm{d} s+\frac{1}{\Gamma(\alpha)} \int_{t}^{1} t^{\alpha-1}(1-s)^{\alpha-1} y(s) \mathrm{d} s \\
& =\int_{0}^{1} G_{1}(\alpha, 0 ; t, s) y(s) \mathrm{d} s .
\end{aligned}
$$

The proof is complete.

Lemma 2.8 If $\alpha-\mu \geq 1$, then the function $G_{1}(\alpha, 0 ; t, s)$ defined by (2.2) satisfies the following conditions:

(i) $G_{1}(\alpha, 0 ; t, s) \geq 0, G_{1}(\alpha, 0 ; t, s) \leq J_{1}(\alpha, 0 ; s)$ for all $t, s \in[0,1]$, where

$$
J_{1}(\alpha, 0 ; s)=\frac{1}{\Gamma(\alpha)}(1-s)^{\alpha-1}
$$


(ii) there exists a positive function $\rho_{1}(s) \in C(0,1)$ such that

$\min _{t \in\left[\gamma_{1}, \delta_{1}\right]} G_{1}(\alpha, 0 ; t, s) \geq \rho_{1}(s) J_{1}(\alpha, 0 ; s), s \in(0,1)$, where $0<\gamma_{1}<\delta_{1}<1 ;$

(iii)

$$
\begin{aligned}
& D_{0^{+}}^{\mu} G_{1}(\alpha, 0 ; t, s) \\
& \quad=\frac{1}{\Gamma(\alpha-\mu)} \begin{cases}t^{\alpha-\mu-1}(1-s)^{\alpha-1}-(t-s)^{\alpha-\mu-1}, & 0 \leq s \leq t \leq 1, \\
t^{\alpha-\mu-1}(1-s)^{\alpha-1}, & 0 \leq t \leq s \leq 1\end{cases}
\end{aligned}
$$

and

$$
D_{0^{+}}^{\mu} G_{1}(\alpha, 0 ; t, s) \leq \frac{1}{\Gamma(\alpha-\mu)}(1-s)^{\alpha-1}=\frac{\Gamma(\alpha)}{\Gamma(\alpha-\mu)} J_{1}(\alpha, 0 ; s) .
$$

Proof On the one hand, by the definition of $G_{1}(\alpha, 0 ; t, s)$, we have

$$
\begin{aligned}
t^{\alpha-1}(1-s)^{\alpha-1}-(t-s)^{\alpha-1} & =t^{\alpha-1}\left[(1-s)^{\alpha-1}-\left(1-\frac{s}{t}\right)^{\alpha-1}\right] \\
& \geq t^{\alpha-1}\left[(1-s)^{\alpha-1}-(1-s)^{\alpha-1}\right] \geq 0,
\end{aligned}
$$

which implies that $G_{1}(\alpha, 0 ; t, s) \geq 0,0 \leq t, s \leq 1$. Obviously, $G_{1}(\alpha, 0 ; t, s) \leq J_{1}(\alpha, 0 ; s)$ for all $t, s \in[0,1]$. On the other hand, let $g_{1}(\alpha, 0 ; t, s)=t^{\alpha-1}(1-s)^{\alpha-1}-(t-s)^{\alpha-1}, 0 \leq s \leq t \leq 1$ and $g_{2}(\alpha, 0 ; t, s)=t^{\alpha-1}(1-s)^{\alpha-1}, 0 \leq t \leq s \leq 1$. Then

$$
\begin{aligned}
\frac{d g_{1}(\alpha, 0 ; t, s)}{d t} & =(\alpha-1)\left[t^{\alpha-2}(1-s)^{\alpha-1}-(t-s)^{\alpha-2}\right] \\
& =(\alpha-1) t^{\alpha-2}\left[(1-s)^{\alpha-1}-\left(1-\frac{s}{t}\right)^{\alpha-2}\right] \\
& \leq(\alpha-1) t^{\alpha-2}\left[(1-s)^{\alpha-1}-(1-s)^{\alpha-2}\right] \leq 0,
\end{aligned}
$$

which implies that $g_{1}(\alpha, 0 ; \cdot, s)$ is nonincreasing for all $s \in(0,1]$. Since $g_{2}(\alpha, 0 ; \cdot, s)$ is nondecreasing for all $s \in(0,1)$, then we have

$$
\begin{aligned}
\min _{t \in\left[\gamma_{1}, \delta_{1}\right]} G_{1}(\alpha, 0 ; t, s) & = \begin{cases}\frac{g_{1}\left(\alpha, 0 ; \delta_{1}, s\right)}{\Gamma(\alpha)}, & s \in\left(0, \gamma_{1}\right], \\
\min \left\{\frac{g_{1}\left(\alpha, 0 ; \delta_{1}, s\right)}{\Gamma(\alpha)}, \frac{g_{2}\left(\alpha, 0 ; \gamma_{1}, s\right)}{\Gamma(\alpha)}\right\}, & s \in\left[\gamma_{1}, \delta_{1}\right], \\
\frac{g_{2}\left(\alpha, 0 ; \gamma_{1}, s\right)}{\Gamma(\alpha)}, & s \in\left[\delta_{1}, 1\right)\end{cases} \\
& = \begin{cases}\frac{g_{1}\left(\alpha, 0 ; \delta_{1}, s\right)}{\Gamma(\alpha)}, & s \in\left(0, r_{1}\right], \\
\frac{g_{2}\left(\alpha, 0 ; \gamma_{1}, s\right)}{\Gamma(\alpha)}, & s \in\left[r_{1}, 1\right)\end{cases} \\
& = \begin{cases}\frac{\delta_{1}^{\alpha-1}(1-s)^{\alpha-1}-\left(\delta_{1}-s\right)^{\alpha-1}}{\Gamma(\alpha)}, & s \in\left(0, r_{1}\right], \\
\frac{\gamma_{1}^{\alpha-1}(1-s)^{\alpha-1}}{\Gamma(\alpha)}, & s \in\left[r_{1}, 1\right),\end{cases}
\end{aligned}
$$

where $\gamma_{1}<r_{1}<\delta_{1}$ is the solution of the equation

$$
\delta_{1}^{\alpha-1}(1-s)^{\alpha-1}-\left(\delta_{1}-s\right)^{\alpha-1}=\gamma_{1}^{\alpha-1}(1-s)^{\alpha-1} .
$$


Thus, we set

$$
\rho_{1}(s)= \begin{cases}\frac{\delta_{1}^{\alpha-1}(1-s)^{\alpha-1}-\left(\delta_{1}-s\right)^{\alpha-1}}{(1-s)^{\alpha-1}}, & s \in\left(0, r_{1}\right] \\ \gamma_{1}^{\alpha-1}, & s \in\left[r_{1}, 1\right)\end{cases}
$$

the proof is complete.

Lemma 2.9 Given $y \in C[0,1]$ and $1<\alpha \leq 2,0 \leq \beta \leq 1, \alpha-\beta-1 \geq 0$, the problem

$$
\left\{\begin{array}{l}
D_{0^{+}}^{\alpha} u(t)+y(t)=0, \quad 0<t<1 \\
u(0)=0, \quad D_{0^{+}}^{\beta} u(1)=0
\end{array}\right.
$$

is equivalent to

$$
u(t)=\int_{0}^{1} G_{2}(\alpha, \beta ; t, s) y(s) \mathrm{d} s
$$

where

$$
G_{2}(\alpha, \beta ; t, s)=\frac{1}{\Gamma(\alpha)} \begin{cases}t^{\alpha-1}(1-s)^{\alpha-\beta-1}-(t-s)^{\alpha-1}, & 0 \leq s \leq t \leq 1 \\ t^{\alpha-1}(1-s)^{\alpha-\beta-1}, & 0 \leq t \leq s \leq 1\end{cases}
$$

Proof In view of Lemma 2.3, the equation $D_{0^{+}}^{\alpha} u(t)+y(t)=0$ is equivalent to the integral equation

$$
u(t)=-\frac{1}{\Gamma(\alpha)} \int_{0}^{t}(t-s)^{\alpha-1} y(s) \mathrm{d} s+c_{1} t^{\alpha-1}+c_{2} t^{\alpha-2}
$$

The boundary condition $u(0)=0$ implies that $c_{2}=0$. Thus

$$
u(t)=-\frac{1}{\Gamma(\alpha)} \int_{0}^{t}(t-s)^{\alpha-1} y(s) \mathrm{d} s+c_{1} t^{\alpha-1}
$$

By Lemma 2.6, we have

$$
D_{0^{+}}^{\beta} u(t)=-\frac{1}{\Gamma(\alpha-\beta)} \int_{0}^{t}(t-s)^{\alpha-\beta-1} y(s) \mathrm{d} s+c_{1} \frac{\Gamma(\alpha)}{\Gamma(\alpha-\beta)} t^{\alpha-\beta-1} .
$$

In view of the boundary condition $D_{0^{+}}^{\beta} u(1)=0$, we conclude that

$$
c_{1}=\frac{1}{\Gamma(\alpha)} \int_{0}^{1}(1-s)^{\alpha-\beta-1} y(s) \mathrm{d} s .
$$

Therefore, the unique solution of problem (2.3) is

$$
u(t)=-\frac{1}{\Gamma(\alpha)} \int_{0}^{t}(t-s)^{\alpha-1} y(s) \mathrm{d} s+\frac{t^{\alpha-1}}{\Gamma(\alpha)} \int_{0}^{1}(1-s)^{\alpha-\beta-1} y(s) \mathrm{d} s
$$


The unique solution of (2.3) is expressed as

$$
\begin{aligned}
u(t) & =\frac{1}{\Gamma(\alpha)} \int_{0}^{t}\left[t^{\alpha-1}(1-s)^{\alpha-\beta-1}-(t-s)^{\alpha-1}\right] y(s) \mathrm{d} s+\frac{1}{\Gamma(\alpha)} \int_{t}^{1} t^{\alpha-1}(1-s)^{\alpha-\beta-1} y(s) \mathrm{d} s \\
& =\int_{0}^{1} G_{2}(\alpha, \beta ; t, s) y(s) \mathrm{d} s .
\end{aligned}
$$

The proof is complete.

Lemma 2.10 If $\alpha-\mu \geq 1, \alpha-\beta \geq 1$, then the function $G_{2}(\alpha, \beta ; t, s)$ defined by (2.4) satisfies the following conditions:

(i) $G_{2}(\alpha, \beta ; t, s) \geq 0, G_{2}(\alpha, \beta ; t, s) \leq J_{2}(\alpha, \beta ; s)$ for all $t, s \in[0,1]$, where $J_{2}(\alpha, \beta ; s)=\frac{1}{\Gamma(\alpha)}(1-s)^{\alpha-\beta-1} ;$

(ii) there exists a positive function $\rho_{2}(s) \in C(0,1)$ such that $\min _{t \in\left[\gamma_{2}, \delta_{2}\right]} G_{2}(\alpha, \beta ; t, s) \geq \rho_{2}(s) J_{2}(\alpha, \beta ; s), s \in(0,1)$, where $0<\gamma_{2}<\delta_{2}<1$;

(iii)

$$
\begin{aligned}
& D_{0^{+}}^{\mu} G_{2}(\alpha, \beta ; t, s) \\
& \quad=\frac{1}{\Gamma(\alpha-\mu)} \begin{cases}t^{\alpha-\mu-1}(1-s)^{\alpha-\beta-1}-(t-s)^{\alpha-\mu-1}, & 0 \leq s \leq t \leq 1, \\
t^{\alpha-\mu-1}(1-s)^{\alpha-\beta-1}, & 0 \leq t \leq s \leq 1\end{cases}
\end{aligned}
$$

and

$$
D_{0^{+}}^{\mu} G_{2}(\alpha, \beta ; t, s) \leq \frac{1}{\Gamma(\alpha-\mu)}(1-s)^{\alpha-\beta-1}=\frac{\Gamma(\alpha)}{\Gamma(\alpha-\mu)} J_{2}(\alpha, \beta ; s) .
$$

Proof On the one hand, by the definition of $G_{2}(\alpha, \beta ; t, s)$, we have

$$
\begin{aligned}
t^{\alpha-1}(1-s)^{\alpha-\beta-1}-(t-s)^{\alpha-1} & =t^{\alpha-1}\left[(1-s)^{\alpha-\beta-1}-\left(1-\frac{s}{t}\right)^{\alpha-1}\right] \\
& \geq t^{\alpha-1}\left[(1-s)^{\alpha-\beta-1}-(1-s)^{\alpha-1}\right] \geq 0
\end{aligned}
$$

which implies that $G_{2}(\alpha, \beta ; t, s) \geq 0,0 \leq t, s \leq 1$. Obviously, $G_{2}(\alpha, \beta ; t, s) \leq J_{2}(\alpha, \beta ; s)$ for all $t, s \in[0,1]$. On the other hand, let $g_{1}(\alpha, \beta ; t, s)=t^{\alpha-1}(1-s)^{\alpha-\beta-1}-(t-s)^{\alpha-1}, 0 \leq s \leq t \leq 1$ and $g_{2}(\alpha, \beta ; t, s)=t^{\alpha-1}(1-s)^{\alpha-\beta-1}, 0 \leq t \leq s \leq 1$. Then

$$
\begin{aligned}
\frac{d g_{1}(\alpha, \beta ; t, s)}{d t} & =(\alpha-1)\left[t^{\alpha-2}(1-s)^{\alpha-\beta-1}-(t-s)^{\alpha-2}\right] \\
& =(\alpha-1) t^{\alpha-2}\left[(1-s)^{\alpha-\beta-1}-\left(1-\frac{s}{t}\right)^{\alpha-2}\right] \\
& \leq(\alpha-1) t^{\alpha-2}\left[(1-s)^{\alpha-\beta-1}-(1-s)^{\alpha-2}\right] \leq 0,
\end{aligned}
$$

which implies that $g_{1}(\alpha, \beta ; \cdot, s)$ is nonincreasing for all $s \in(0,1]$. Since $g_{2}(\alpha, \beta ; \cdot, s)$ is nondecreasing for all $s \in(0,1)$, then we have

$$
\min _{t \in\left[\gamma_{2}, \delta_{2}\right]} G_{2}(\alpha, \beta ; t, s)= \begin{cases}\frac{g_{1}\left(\alpha, \beta ; \delta_{2}, s\right)}{\Gamma(\alpha)}, & s \in\left(0, \gamma_{2}\right], \\ \min \left\{\frac{g_{1}\left(\alpha, \beta ; \delta_{2}, s\right)}{\Gamma(\alpha)}, \frac{g_{2}\left(\alpha, \beta ; \gamma_{2}, s\right)}{\Gamma(\alpha)}\right\}, & s \in\left[\gamma_{2}, \delta_{2}\right], \\ \frac{g_{2}(\alpha, \beta ; \gamma, s)}{\Gamma(\alpha)}, & s \in\left[\delta_{2}, 1\right)\end{cases}
$$




$$
\begin{aligned}
& = \begin{cases}\frac{g_{1}\left(\alpha, \beta ; \delta_{2}, s\right)}{\Gamma(\alpha)}, & s \in\left(0, r_{2}\right], \\
\frac{g_{2}\left(\alpha, \beta ; \gamma_{2}, s\right)}{\Gamma(\alpha)}, & s \in\left[r_{2}, 1\right)\end{cases} \\
& = \begin{cases}\frac{\delta_{2}^{\alpha-1}(1-s)^{\alpha-\beta-1}-\left(\delta_{2}-s\right)^{\alpha-1}}{\Gamma(\alpha)}, & s \in\left(0, r_{2}\right], \\
\frac{\gamma_{2}^{\alpha-1}(1-s)^{\alpha-\beta-1}}{\Gamma(\alpha)}, & s \in\left[r_{2}, 1\right),\end{cases}
\end{aligned}
$$

where $\gamma_{2}<r_{2}<\delta_{2}$ is the solution of equation

$$
\delta_{2}^{\alpha-1}(1-s)^{\alpha-\beta-1}-\left(\delta_{2}-s\right)^{\alpha-1}=\gamma_{2}^{\alpha-1}(1-s)^{\alpha-\beta-1} .
$$

Thus, we set

$$
\rho_{2}(s)= \begin{cases}\frac{\delta_{2}^{\alpha-1}(1-s)^{\alpha-\beta-1}-\left(\delta_{2}-s\right)^{\alpha-1}}{(1-s)^{\alpha-\beta-1}}, & s \in\left(0, r_{2}\right], \\ \gamma_{2}^{\alpha-1}, & s \in\left[r_{2}, 1\right),\end{cases}
$$

the proof is complete.

Lemma 2.11 Given $y \in C[0,1]$ and $1<\alpha \leq 2,0 \leq \beta \leq 1$, if $\Delta=1-k \xi^{\alpha-1}+\frac{\Gamma(\alpha)}{\Gamma(\alpha-\beta)}(1-$ $\left.l \eta^{\alpha-\beta-1}\right) \neq 0$, the problem

$$
\left\{\begin{array}{l}
D_{0^{+}}^{\alpha} u(t)+y(t)=0, \quad 0<t<1, \\
u(0)=0, \quad u(1)+D_{0^{+}}^{\beta} u(1)=k u(\xi)+l D_{0^{+}}^{\beta} u(\eta),
\end{array}\right.
$$

is equivalent to

$$
u(t)=\int_{0}^{1} G(t, s) y(s) \mathrm{d} s
$$

where

$$
\begin{aligned}
G(t, s)= & \frac{1}{\Delta}\left[\left(1-k \xi^{\alpha-1}\right) G_{1}(\alpha, 0 ; t, s)+k t^{\alpha-1} G_{1}(\alpha, 0 ; \xi, s)\right. \\
& \left.+\frac{\Gamma(\alpha)\left(1-l \eta^{\alpha-\beta-1}\right)}{\Gamma(\alpha-\beta)} G_{2}(\alpha, \beta ; t, s)+l t^{\alpha-1} G_{1}(\alpha-\beta, 0 ; \eta, s)\right] .
\end{aligned}
$$

Proof In view of Lemma 2.3, the equation $D_{0^{+}}^{\alpha} u(t)+y(t)=0$ is equivalent to the integral equation

$$
u(t)=-\frac{1}{\Gamma(\alpha)} \int_{0}^{t}(t-s)^{\alpha-1} y(s) \mathrm{d} s+c_{1} t^{\alpha-1}+c_{2} t^{\alpha-2}
$$

The boundary condition $u(0)=0$ implies that $c_{2}=0$. Thus

$$
u(t)=-\frac{1}{\Gamma(\alpha)} \int_{0}^{t}(t-s)^{\alpha-1} y(s) \mathrm{d} s+c_{1} t^{\alpha-1}
$$

By Lemma 2.6, we have

$$
D_{0^{+}}^{\beta} u(t)=-\frac{1}{\Gamma(\alpha-\beta)} \int_{0}^{t}(t-s)^{\alpha-\beta-1} y(s) \mathrm{d} s+c_{1} \frac{\Gamma(\alpha)}{\Gamma(\alpha-\beta)} t^{\alpha-\beta-1} .
$$


In view of the boundary condition $u(1)+D_{0^{+}}^{\beta} u(1)=k u(\xi)+l D_{0^{+}}^{\beta} u(\eta)$, we conclude that

$$
\begin{aligned}
c_{1}= & \frac{1}{\Delta}\left[\frac{1}{\Gamma(\alpha)} \int_{0}^{1}(1-s)^{\alpha-1} y(s) \mathrm{d} s-\frac{k}{\Gamma(\alpha)} \int_{0}^{\xi}(\xi-s)^{\alpha-1} y(s) \mathrm{d} s\right. \\
& \left.+\frac{1}{\Gamma(\alpha-\beta)} \int_{0}^{1}(1-s)^{\alpha-\beta-1} y(s) \mathrm{d} s-\frac{l}{\Gamma(\alpha-\beta)} \int_{0}^{\eta}(\eta-s)^{\alpha-\beta-1} y(s) \mathrm{d} s\right] .
\end{aligned}
$$

Therefore, the unique solution of problem (2.5) is

$$
\begin{aligned}
u(t)= & -\frac{1}{\Gamma(\alpha)} \int_{0}^{t}(t-s)^{\alpha-1} y(s) \mathrm{d} s \\
& +\frac{t^{\alpha-1}}{\Delta}\left[\frac{1}{\Gamma(\alpha)} \int_{0}^{1}(1-s)^{\alpha-1} y(s) \mathrm{d} s-\frac{k}{\Gamma(\alpha)} \int_{0}^{\xi}(\xi-s)^{\alpha-1} y(s) \mathrm{d} s\right. \\
& \left.+\frac{1}{\Gamma(\alpha-\beta)} \int_{0}^{1}(1-s)^{\alpha-\beta-1} y(s) \mathrm{d} s-\frac{l}{\Gamma(\alpha-\beta)} \int_{0}^{\eta}(\eta-s)^{\alpha-\beta-1} y(s) \mathrm{d} s\right] .
\end{aligned}
$$

The unique solution of (2.5) is expressed as

$$
\begin{aligned}
u(t)= & \frac{1}{\Delta}\left[\frac{1-k \xi^{\alpha-1}}{\Gamma(\alpha)}\left(\int_{0}^{t}\left[t^{\alpha-1}(1-s)^{\alpha-1}-(t-s)^{\alpha-1}\right] y(s) \mathrm{d} s+\int_{t}^{1} t^{\alpha-1}(1-s)^{\alpha-1} y(s) \mathrm{d} s\right)\right. \\
& +\frac{k t^{\alpha-1}}{\Gamma(\alpha)}\left(\int_{0}^{\xi}\left[\xi^{\alpha-1}(1-s)^{\alpha-1}-(\xi-s)^{\alpha-1}\right] y(s) \mathrm{d} s+\int_{\xi}^{1} \xi^{\alpha-1}(1-s)^{\alpha-1} y(s) \mathrm{d} s\right) \\
& +\frac{1-l \eta^{\alpha-\beta-1}}{\Gamma(\alpha-\beta)}\left(\int_{0}^{t}\left[t^{\alpha-1}(1-s)^{\alpha-\beta-1}-(t-s)^{\alpha-1}\right] y(s) \mathrm{d} s\right. \\
& \left.+\int_{t}^{1} t^{\alpha-1}(1-s)^{\alpha-\beta-1} y(s) \mathrm{d} s\right) \\
& +\frac{l t^{\alpha-1}}{\Gamma(\alpha-\beta)}\left(\int_{0}^{\eta}\left[\eta^{\alpha-\beta-1}(1-s)^{\alpha-\beta-1}-(\eta-s)^{\alpha-\beta-1}\right] y(s) \mathrm{d} s\right. \\
& \left.\left.+\int_{\eta}^{1} \eta^{\alpha-\beta-1}(1-s)^{\alpha-\beta-1} y(s) \mathrm{d} s\right)\right] \\
= & \frac{1}{\Delta}\left[\left(1-k \xi^{\alpha-1}\right) \int_{0}^{1} G_{1}(\alpha, 0 ; t, s) y(s) \mathrm{d} s+k t^{\alpha-1} \int_{0}^{1} G_{1}(\alpha, 0 ; \xi, s) y(s) \mathrm{d} s\right. \\
& +\frac{\Gamma(\alpha)\left(1-l \eta^{\alpha-\beta-1}\right)}{\Gamma(\alpha-\beta)} \int_{0}^{1} G_{2}(\alpha, \beta ; t, s) y(s) \mathrm{d} s \\
& \left.+l t^{\alpha-1} \int_{0}^{1} G_{1}(\alpha-\beta, 0 ; \eta, s) y(s) \mathrm{d} s\right] .
\end{aligned}
$$

The proof is complete.

Lemma 2.12 If condition $\left(\mathrm{H}_{1}\right)$ holds, then the function $G(t, s)$ defined by (2.6) satisfies the following conditions:

(i) $G(t, s) \geq 0, G(t, s) \leq J(s)$ for all $t, s \in[0,1]$, where

$$
\begin{aligned}
J(s)= & \frac{1}{\Delta}\left[\left(1-k \xi^{\alpha-1}\right) J_{1}(\alpha, 0 ; s)+k G_{1}(\alpha, 0 ; \xi, s)\right. \\
& \left.+\frac{\Gamma(\alpha)\left(1-l \eta^{\alpha-\beta-1}\right)}{\Gamma(\alpha-\beta)} J_{2}(\alpha, \beta ; s)+l G_{1}(\alpha-\beta, 0 ; \eta, s)\right]
\end{aligned}
$$


(ii) there exists a positive function $\rho(s) \in C(0,1)$ such that $\min _{t \in[\gamma, \delta]} G(t, s) \geq \rho(s) J(s)$, $s \in(0,1)$, where $0<\gamma<\delta<1$.

Proof (i) From Lemma 2.8 and Lemma 2.10, we get $G(t, s) \geq 0$ and

$$
\begin{aligned}
G(t, s) \leq & \frac{1}{\Delta}\left[\left(1-k \xi^{\alpha-1}\right) J_{1}(\alpha, 0 ; s)+k G_{1}(\alpha, 0 ; \xi, s)\right. \\
& \left.+\frac{\Gamma(\alpha)\left(1-l \eta^{\alpha-\beta-1}\right)}{\Gamma(\alpha-\beta)} J_{2}(\alpha, \beta ; s)+l G_{1}(\alpha-\beta, 0 ; \eta, s)\right] \\
= & J(s) .
\end{aligned}
$$

(ii) From Lemma 2.8 and Lemma 2.10, for $t \in[\gamma, \delta]$, we have

$$
\begin{aligned}
G(t, s)= & \frac{1}{\Delta}\left[\left(1-k \xi^{\alpha-1}\right) G_{1}(\alpha, 0 ; t, s)+k t^{\alpha-1} G_{1}(\alpha, 0 ; \xi, s)\right. \\
& \left.+\frac{\Gamma(\alpha)\left(1-l \eta^{\alpha-\beta-1}\right)}{\Gamma(\alpha-\beta)} G_{2}(\alpha, \beta ; t, s)+l t^{\alpha-1} G_{1}(\alpha-\beta, 0 ; \eta, s)\right] \\
\geq & \frac{1}{\Delta}\left[\left(1-k \xi^{\alpha-1}\right) \rho_{1}(s) J_{1}(\alpha, 0 ; s)+k \gamma^{\alpha-1} G_{1}(\alpha, 0 ; \xi, s)\right. \\
& \left.+\frac{\Gamma(\alpha)\left(1-l \eta^{\alpha-\beta-1}\right)}{\Gamma(\alpha-\beta)} \rho_{2}(s) J_{2}(\alpha, \beta ; s)+l \gamma^{\alpha-1} G_{1}(\alpha-\beta, 0 ; \eta, s)\right] \\
\geq & \frac{\rho(s)}{\Delta}\left[\left(1-k \xi^{\alpha-1}\right) J_{1}(\alpha, 0 ; s)+k G_{1}(\alpha, 0 ; \xi, s)\right. \\
& \left.+\frac{\Gamma(\alpha)\left(1-l \eta^{\alpha-\beta-1}\right)}{\Gamma(\alpha-\beta)} J_{2}(\alpha, \beta ; s)+l G_{1}(\alpha-\beta, 0 ; \eta, s)\right] \\
= & \rho(s) J(s),
\end{aligned}
$$

where $\rho(s)=\min \left\{\rho_{1}(s), \rho_{2}(s), \gamma^{\alpha-1}\right\}, \gamma=\max \left\{\gamma_{1}, \gamma_{2}\right\}, \delta=\min \left\{\delta_{1}, \delta_{2}\right\}$. The proof is complete.

\section{Main results}

We define the space $E=\left\{u(t) \mid u(t) \in C[0,1]\right.$ and $\left.D_{0^{+}}^{\mu} u(t) \in C[0,1]\right\}$ is endowed with the ordering $u \leq v$ if $u(t) \leq v(t)$ for all $t \in[0,1]$, and endowed with the norm $\|u\|=$ $\max \left\{\|u\|_{0},\left\|D_{0^{+}}^{\mu} u\right\|_{0}\right\}$, where $\|u\|_{0}=\max _{t \in[0,1]}|u(t)|$.

Lemma $3.1(E,\|\cdot\|)$ is a Banach space.

Proof Let $\left\{u_{n}\right\}_{n=1}^{\infty}$ be a Cauchy sequence in the space $(E,\|\cdot\|)$. Then clearly $\left\{u_{n}\right\}_{n=1}^{\infty}$ and $\left\{D_{0^{+}}^{\mu} u_{n}\right\}_{n=1}^{\infty}$ are Cauchy sequences in the space $C[0,1]$. Therefore, $\left\{u_{n}\right\}_{n=1}^{\infty}$ and $\left\{D_{0^{+}}^{\mu} u_{n}\right\}_{n=1}^{\infty}$ converge to some $v$ and $w$ on $[0,1]$ uniformly and $v, w \in C[0,1]$. We need to prove that $w=D_{0^{+}}^{\mu} v$.

Note that

$$
\begin{aligned}
\left|I_{0^{+}}^{\mu} D_{0^{+}}^{\mu} u_{n}(t)-I_{0^{+}}^{\mu} w(t)\right| & \leq \frac{1}{\Gamma(\mu)} \int_{0}^{t}(t-s)^{\mu-1}\left|D_{0^{+}}^{\mu} u_{n}(s)-w(s)\right| \mathrm{d} s \\
& \leq \frac{1}{\Gamma(\mu+1)} \max _{t \in[0,1]}\left|D_{0^{+}}^{\mu} u_{n}(t)-w(t)\right| .
\end{aligned}
$$


By the convergence of $\left\{D_{0^{+}}^{\mu} u_{n}\right\}_{n=1}^{\infty}$, we have $\lim _{n \rightarrow \infty} I_{0^{+}}^{\mu} D_{0^{+}}^{\mu} u_{n}(t)=I_{0^{+}}^{\mu} w(t)$ uniformly for $t \in[0,1]$. On the other hand, by Remark 2.5 one has $I_{0^{+}}^{\mu} D_{0^{+}}^{\mu} u_{n}(t)=u_{n}(t)$. Hence, $v(t)=$ $I_{0^{+}}^{\mu} w(t)$, Remark 2.5 implies that it is equivalent to the relation $w=D_{0^{+}}^{\mu} \nu$. This completes the proof.

Define the cone $P \subset E$ by

$$
P=\{u \in E \mid u(t) \geq 0\}
$$

Let the nonnegative continuous concave functional $\theta$ on the cone $P$ be defined by

$$
\theta(u)=\min _{\gamma \leq t \leq \delta}|u(t)| .
$$

Lemma 3.2 If conditions $\left(\mathrm{H}_{1}\right),\left(\mathrm{H}_{2}\right)$ hold. Let $T: P \rightarrow E$ be the operator defined by

$$
\operatorname{Tu}(t):=\int_{0}^{1} G(t, s) f\left(s, u(s), D_{0^{+}}^{\mu} u(s)\right) \mathrm{d} s, \quad 0 \leq t \leq 1,
$$

then $T: P \rightarrow P$ is completely continuous.

Proof The operator $T: P \rightarrow P$ is continuous in view of the non-negativeness and continuity of $G(t, s)$ and $f\left(t, u(t), D_{0^{+}}^{\mu} u(t)\right)$.

Let $\Omega \subset P$ be bounded, i.e., there exists a positive constant $M>0$ such that $\|u\| \leq M$ for all $u \in \Omega$. Let $L=\max _{0 \leq t \leq 1,0 \leq u \leq M,-M \leq D_{0^{+}}^{\mu} u \leq M}\left|f\left(t, u(t), D_{0^{+}}^{\mu} u(t)\right)\right|+1$, then, for $u \in \Omega$, we have

$$
\begin{array}{rl}
|T u(t)|=\int_{0}^{1} & G(t, s) f\left(s, u(s), D_{0^{+}}^{\mu} u(s)\right) \mathrm{d} s \leq L \int_{0}^{1} J(s) \mathrm{d} s \\
\left|D_{0^{+}}^{\mu} T u(t)\right|= & \frac{1}{\Delta}\left[\left(1-k \xi^{\alpha-1}\right) \int_{0}^{1} D_{0^{+}}^{\mu} G_{1}(\alpha, 0 ; t, s) f\left(s, u(s), D_{0^{+}}^{\mu} u(s)\right) \mathrm{d} s\right. \\
& +k t^{\alpha-\mu-1} \frac{\Gamma(\alpha)}{\Gamma(\alpha-\mu)} \int_{0}^{1} G_{1}(\alpha, 0 ; \xi, s) f\left(s, u(s), D_{0^{+}}^{\mu} u(s)\right) \mathrm{d} s \\
& +\frac{\Gamma(\alpha)\left(1-l \eta^{\alpha-\beta-1}\right)}{\Gamma(\alpha-\beta)} \int_{0}^{1} D_{0^{+}}^{\mu} G_{2}(\alpha, \beta ; t, s) f\left(s, u(s), D_{0^{+}}^{\mu} u(s)\right) \mathrm{d} s \\
& \left.+l t^{\alpha-\mu-1} \frac{\Gamma(\alpha)}{\Gamma(\alpha-\mu)} \int_{0}^{1} G_{1}(\alpha-\beta, 0 ; \eta, s) f\left(s, u(s), D_{0^{+}}^{\mu} u(s)\right) \mathrm{d} s\right] \\
\leq & \frac{L}{\Delta}\left[\left(1-k \xi^{\alpha-1}\right) \frac{\Gamma(\alpha)}{\Gamma(\alpha-\mu)} \int_{0}^{1} J_{1}(\alpha, 0 ; s) \mathrm{d} s+k \frac{\Gamma(\alpha)}{\Gamma(\alpha-\mu)} \int_{0}^{1} G_{1}(\alpha, 0 ; \xi, s) \mathrm{d} s\right. \\
& +\frac{\Gamma(\alpha)\left(1-l \eta^{\alpha-\beta-1}\right)}{\Gamma(\alpha-\beta)} \frac{\Gamma(\alpha)}{\Gamma(\alpha-\mu)} \int_{0}^{1} J_{2}(\alpha, \beta ; s) \mathrm{d} s \\
& \left.+l \frac{\Gamma(\alpha)}{\Gamma(\alpha-\mu)} \int_{0}^{1} G_{1}(\alpha-\beta, 0 ; \eta, s) \mathrm{d} s\right] \\
\leq & \frac{\Gamma(\alpha)}{\Gamma(\alpha-\mu)} L \int_{0}^{1} J(s) \mathrm{d} s .
\end{array}
$$

Hence, $T(\Omega)$ is bounded. 
On the other hand, given $\varepsilon>0$, set $\delta=\frac{1}{2}\left(\frac{\Delta \varepsilon}{L Q}\right)^{\frac{1}{\alpha-1}}$, where

$$
Q=\frac{1-k \xi^{\alpha-1}}{\Gamma(\alpha)}+k \int_{0}^{1} G_{1}(\alpha, 0 ; \xi, s) \mathrm{d} s+\frac{1-l \eta^{\alpha-\beta-1}}{\Gamma(\alpha-\beta)}+l \int_{0}^{1} G_{1}(\alpha-\beta, 0 ; \eta, s) \mathrm{d} s,
$$

then, for each $u \in \Omega, t_{1}, t_{2} \in[0,1], t_{1}<t_{2}$, and $t_{2}-t_{1}<\delta$, one has $\left|T u\left(t_{2}\right)-T u\left(t_{1}\right)\right|<\varepsilon$. That is to say, $T(\Omega)$ is equicontinuous.

In fact,

$$
\begin{aligned}
& \left|T u\left(t_{2}\right)-T u\left(t_{1}\right)\right| \\
& =\left|\int_{0}^{1} G\left(t_{2}, s\right) f\left(s, u(s), D_{0^{+}}^{\mu} u(s)\right) \mathrm{d} s-\int_{0}^{1} G\left(t_{1}, s\right) f\left(s, u(s), D_{0^{+}}^{\mu} u(s)\right) \mathrm{d} s\right| \\
& =\frac{1}{\Delta} \mid\left(1-k \xi^{\alpha-1}\right)\left(\int_{0}^{1} G_{1}\left(\alpha, 0 ; t_{2}, s\right) f\left(s, u(s), D_{0^{+}}^{\mu} u(s)\right) \mathrm{d} s\right. \\
& \left.-\int_{0}^{1} G_{1}\left(\alpha, 0 ; t_{1}, s\right) f\left(s, u(s), D_{0^{+}}^{\mu} u(s)\right) \mathrm{d} s\right) \\
& +k\left(t_{2}^{\alpha-1}-t_{1}^{\alpha-1}\right) \int_{0}^{1} G_{1}(\alpha, 0 ; \xi, s) f\left(s, u(s), D_{0^{+}}^{\mu} u(s)\right) \mathrm{d} s \\
& +\frac{\Gamma(\alpha)\left(1-l \eta^{\alpha-\beta-1}\right)}{\Gamma(\alpha-\beta)}\left(\int_{0}^{1} G_{2}\left(\alpha, \beta ; t_{2}, s\right) f\left(s, u(s), D_{0^{+}}^{\mu} u(s)\right) \mathrm{d} s\right. \\
& \left.-\int_{0}^{1} G_{2}\left(\alpha, \beta ; t_{1}, s\right) f\left(s, u(s), D_{0^{+}}^{\mu} u(s)\right) \mathrm{d} s\right) \\
& +l\left(t_{2}^{\alpha-1}-t_{1}^{\alpha-1}\right) \int_{0}^{1} G_{1}(\alpha-\beta, 0 ; \eta, s) f\left(s, u(s), D_{0^{+}}^{\mu} u(s)\right) \mathrm{d} s \mid \\
& =\frac{1}{\Delta} \mid\left(1-k \xi^{\alpha-1}\right) \int_{0}^{1}\left(G_{1}\left(\alpha, 0 ; t_{2}, s\right)-G_{1}\left(\alpha, 0 ; t_{1}, s\right)\right) f\left(s, u(s), D_{0^{+}}^{\mu} u(s)\right) \mathrm{d} s \\
& +k\left(t_{2}^{\alpha-1}-t_{1}^{\alpha-1}\right) \int_{0}^{1} G_{1}(\alpha, 0 ; \xi, s) f\left(s, u(s), D_{0^{+}}^{\mu} u(s)\right) \mathrm{d} s \\
& +\frac{\Gamma(\alpha)\left(1-l \eta^{\alpha-\beta-1}\right)}{\Gamma(\alpha-\beta)} \int_{0}^{1}\left(G_{2}\left(\alpha, \beta ; t_{2}, s\right)-G_{2}\left(\alpha, \beta ; t_{1}, s\right)\right) f\left(s, u(s), D_{0^{+}}^{\mu} u(s)\right) \mathrm{d} s \\
& +l\left(t_{2}^{\alpha-1}-t_{1}^{\alpha-1}\right) \int_{0}^{1} G_{1}(\alpha-\beta, 0 ; \eta, s) f\left(s, u(s), D_{0^{+}}^{\mu} u(s)\right) \mathrm{d} s \\
& \leq \frac{L}{\Delta}\left[\frac { 1 - k \xi ^ { \alpha - 1 } } { \Gamma ( \alpha ) } \left(\int_{0}^{t_{1}}\left(t_{2}^{\alpha-1}-t_{1}^{\alpha-1}\right)(1-s)^{\alpha-1} \mathrm{~d} s+\int_{t_{2}}^{1}\left(t_{2}^{\alpha-1}-t_{1}^{\alpha-1}\right)(1-s)^{\alpha-1} \mathrm{~d} s\right.\right. \\
& \left.+\int_{t_{1}}^{t_{2}}\left(t_{2}^{\alpha-1}-t_{1}^{\alpha-1}\right)(1-s)^{\alpha-1} \mathrm{~d} s\right)+k\left(t_{2}^{\alpha-1}-t_{1}^{\alpha-1}\right) \int_{0}^{1} G_{1}(\alpha, 0 ; \xi, s) \mathrm{d} s \\
& +\frac{1-l \eta^{\alpha-\beta-1}}{\Gamma(\alpha-\beta)}\left(\int_{0}^{t_{1}}\left(t_{2}^{\alpha-1}-t_{1}^{\alpha-1}\right)(1-s)^{\alpha-\beta-1} \mathrm{~d} s+\int_{t_{2}}^{1}\left(t_{2}^{\alpha-1}-t_{1}^{\alpha-1}\right)(1-s)^{\alpha-\beta-1} \mathrm{~d} s\right. \\
& \left.\left.+\int_{t_{1}}^{t_{2}}\left(t_{2}^{\alpha-1}-t_{1}^{\alpha-1}\right)(1-s)^{\alpha-\beta-1} \mathrm{~d} s\right)+l\left(t_{2}^{\alpha-1}-t_{1}^{\alpha-1}\right) \int_{0}^{1} G_{1}(\alpha-\beta, 0 ; \eta, s) \mathrm{d} s\right] \\
& <\frac{L}{\Delta}\left[\frac{1-k \xi^{\alpha-1}}{\Gamma(\alpha)}+k \int_{0}^{1} G_{1}(\alpha, 0 ; \xi, s) \mathrm{d} s+\frac{1-l \eta^{\alpha-\beta-1}}{\Gamma(\alpha-\beta)}\right.
\end{aligned}
$$




$$
\begin{aligned}
& \left.+l \int_{0}^{1} G_{1}(\alpha-\beta, 0 ; \eta, s) \mathrm{d} s\right]\left(t_{2}^{\alpha-1}-t_{1}^{\alpha-1}\right) \\
= & \frac{L Q}{\Delta}\left(t_{2}^{\alpha-1}-t_{1}^{\alpha-1}\right) .
\end{aligned}
$$

In the following, we divide the proof into two cases.

Case 1. $\delta \leq t_{1}<t_{2}<1$,

$$
\left|T u\left(t_{2}\right)-T u\left(t_{1}\right)\right|<\frac{L Q}{\Delta}\left(t_{2}^{\alpha-1}-t_{1}^{\alpha-1}\right) \leq \frac{L Q}{\Delta} \frac{\alpha-1}{\delta^{2-\alpha}}\left(t_{2}-t_{1}\right) \leq \frac{L Q}{\Delta}(\alpha-1) \delta^{\alpha-1} \leq \varepsilon .
$$

Case $2.0 \leq t_{1}<\delta, t_{2}<2 \delta$,

$$
\left|T u\left(t_{2}\right)-T u\left(t_{1}\right)\right|<\frac{L Q}{\Delta}\left(t_{2}^{\alpha-1}-t_{1}^{\alpha-1}\right) \leq \frac{L Q}{\Delta} t_{2}^{\alpha-1} \leq \frac{L Q}{\Delta}(2 \delta)^{\alpha-1} \leq \varepsilon .
$$

By means of the Arzela-Ascoli theorem, we have $T: P \rightarrow P$ is completely continuous. The proof is complete.

For convenience, we denote

$$
M=\frac{1}{\max \left\{\frac{\Gamma(\alpha)}{\Gamma(\alpha-\mu)} \int_{0}^{1} J(s) \mathrm{d} s, \int_{0}^{1} J(s) \mathrm{d} s\right\}}, \quad N=\frac{1}{\int_{\gamma}^{\delta} \rho(s) J(s) \mathrm{d} s} .
$$

Theorem 3.3 Assume that conditions $\left(\mathrm{H}_{1}\right),\left(\mathrm{H}_{2}\right)$ hold. In addition assume that there exist nonnegative numbers $0<a<b<\frac{b}{\gamma}<\frac{c}{\max \{1, \Gamma(\mu+1)\}}$ such that $\max \{1, \Gamma(\mu+1)\} \cdot b<c \gamma$, and $f(t, u, v)$ satisfies the following growth conditions:

$\left(\mathrm{H}_{3}\right) f(t, u, v) \leq M c$ for $(t, u, v) \in[0,1] \times[0, c] \times[-c, c]$;

$\left(\mathrm{H}_{4}\right) f(t, u, v)<$ Ma for $(t, u, v) \in[0,1] \times[0, a] \times[-a, a]$;

$\left(\mathrm{H}_{5}\right) f(t, u, v) \geq N b$ for $(t, u, v) \in[\gamma, \delta] \times[b, c] \times[-c, c]$.

Then BVP (1.1) has at least three positive solutions $u_{1}, u_{2}$ and $u_{3}$, which satisfy

$$
\begin{aligned}
& \left\|u_{1}(t)\right\|<a, \quad b<\theta\left(u_{2}(t)\right)<\left\|u_{2}(t)\right\| \leq c \quad \text { and } \\
& a<\left\|u_{3}(t)\right\| \leq c \quad \text { with } \theta\left(u_{3}(t)\right)<b .
\end{aligned}
$$

Proof We show that all the conditions of Theorem 1.1 are satisfied.

If $u \in \bar{P}_{c}$, then $\|u\| \leq c$. So $0 \leq u(t) \leq c,-c \leq D_{0^{+}}^{\mu} u(t) \leq c, t \in[0,1]$. Condition $\left(\mathrm{H}_{3}\right)$ implies $f\left(t, u(t), D_{0^{+}}^{\mu} u(t)\right) \leq M c$ for $0 \leq t \leq 1$. Consequently,

$$
\begin{aligned}
\|T u\|= & \max _{0 \leq t \leq 1}\left|\int_{0}^{1} G(t, s) f\left(s, u(s), D_{0^{+}}^{\mu} u(s)\right) \mathrm{d} s\right| \\
\leq & \int_{0}^{1} J(s) f\left(s, u(s), D_{0^{+}}^{\mu} u(s)\right) \mathrm{d} s \leq M c \int_{0}^{1} J(s) \mathrm{d} s \leq c, \\
D_{0^{+}}^{\mu} T u= & \frac{1}{\Delta}\left[\left(1-k \xi^{\alpha-1}\right) \int_{0}^{1} D_{0^{+}}^{\mu} G_{1}(\alpha, 0 ; t, s) f\left(s, u(s), D_{0^{+}}^{\mu} u(s)\right) \mathrm{d} s\right. \\
& +k t^{\alpha-\mu-1} \frac{\Gamma(\alpha)}{\Gamma(\alpha-\mu)} \int_{0}^{1} G_{1}(\alpha, 0 ; \xi, s) f\left(s, u(s), D_{0^{+}}^{\mu} u(s)\right) \mathrm{d} s
\end{aligned}
$$




$$
\begin{aligned}
& +\frac{\Gamma(\alpha)\left(1-l \eta^{\alpha-\beta-1}\right)}{\Gamma(\alpha-\beta)} \int_{0}^{1} D_{0^{+}}^{\mu} G_{2}(\alpha, \beta ; t, s) f\left(s, u(s), D_{0^{+}}^{\mu} u(s)\right) \mathrm{d} s \\
& \left.+l t^{\alpha-\mu-1} \frac{\Gamma(\alpha)}{\Gamma(\alpha-\mu)} \int_{0}^{1} G_{1}(\alpha-\beta, 0 ; \eta, s) f\left(s, u(s), D_{0^{+}}^{\mu} u(s)\right) \mathrm{d} s\right] \\
& \leq \frac{1}{\Delta}\left[\left(1-k \xi^{\alpha-1}\right) \frac{\Gamma(\alpha)}{\Gamma(\alpha-\mu)} \int_{0}^{1} J_{1}(\alpha, 0 ; s) M c \mathrm{~d} s\right. \\
& +k \frac{\Gamma(\alpha)}{\Gamma(\alpha-\mu)} \int_{0}^{1} G_{1}(\alpha, 0 ; \xi, s) M c \mathrm{~d} s \\
& +\frac{\Gamma(\alpha)\left(1-l \eta^{\alpha-\beta-1}\right)}{\Gamma(\alpha-\beta)} \frac{\Gamma(\alpha)}{\Gamma(\alpha-\mu)} \int_{0}^{1} J_{2}(\alpha, \beta ; s) M c \mathrm{~d} s \\
& \left.+l \frac{\Gamma(\alpha)}{\Gamma(\alpha-\mu)} \int_{0}^{1} G_{1}(\alpha-\beta, 0 ; \eta, s) M c \mathrm{~d} s\right] \\
& \leq \frac{\Gamma(\alpha)}{\Gamma(\alpha-\mu)} M c \int_{0}^{1} J(s) \mathrm{d} s \leq c .
\end{aligned}
$$

Therefore, $T: \bar{P}_{c} \rightarrow \bar{P}_{c}$. Standard applications of the Arzela-Ascoli theorem imply that $T$ is a completely continuous operator.

In a completely analogous argument, condition $\left(\mathrm{H}_{4}\right)$ implies that condition $\left(\mathrm{C}_{2}\right)$ of Theorem 1.1 is satisfied.

To check condition $\left(C_{1}\right)$ of Theorem 1.1 , we choose $u(t)=\frac{b}{\gamma} t^{\mu}, 0 \leq t \leq 1$. It is easy to see that

$$
\begin{aligned}
& \theta(u(t))=\min _{\gamma \leq t \leq \delta}\left(\frac{b}{\gamma} t^{\mu}\right) \geq \frac{b}{\gamma} \gamma^{\mu}>b, \\
& \|u\|=\max \left\{\max _{t \in[0,1]}|u(t)|, \max _{t \in[0,1]}\left|D_{0^{+}}^{\mu} u(t)\right|\right\}=\max \left\{\frac{b}{\gamma}, \Gamma(\mu+1) \frac{b}{\gamma}\right\} \leq c .
\end{aligned}
$$

Consequently, $\{u \in P(\theta, b, c) \mid \theta(u)>b\} \neq \emptyset$. Hence, if $u \in P(\theta, b, c)$, then $b \leq u(t) \leq c,-c \leq$ $D_{0^{+}}^{\mu} u(t) \leq c$ for $\gamma \leq t \leq \delta$. From condition $\left(\mathrm{H}_{5}\right)$, we have $f\left(t, u(t), D_{0^{+}}^{\mu} u(t)\right) \geq N b$ for $\gamma \leq$ $t \leq \delta$. So

$$
\begin{aligned}
\theta(\operatorname{Tu}(t)) & =\min _{\gamma \leq t \leq \delta}|T u(t)| \\
& \geq \int_{0}^{1} \rho(s) J(s) f\left(s, u(s), D_{0^{+}}^{\mu} u(s)\right) \mathrm{d} s>N b \int_{\gamma}^{\delta} \rho(s) J(s) \mathrm{d} s \geq b,
\end{aligned}
$$

i.e., $\theta(T u)>b$ for all $u \in P(\theta, b, c)$. This shows that condition $\left(\mathrm{C}_{1}\right)$ of Theorem 1.1 is also satisfied.

By Theorem 1.1 and Remark 1.2, BVP (1.1) has at least three positive solutions $u_{1}, u_{2}$ and $u_{3}$, which satisfy

$$
\begin{aligned}
& \left\|u_{1}(t)\right\|<a, \quad b<\theta\left(u_{2}(t)\right)<\left\|u_{2}(t)\right\| \leq c \quad \text { and } \\
& a<\left\|u_{3}(t)\right\| \leq c \quad \text { with } \theta\left(u_{3}(t)\right)<b .
\end{aligned}
$$

The proof is complete. 


\section{Competing interests}

The authors declare that they have no competing interests.

\section{Authors' contributions}

All authors contributed equally to the manuscript and read and approved the final draft.

\section{Author details}

${ }^{1}$ College of Sciences, Hebei University of Science and Technology, Shijiazhuang, Hebei 050018, P.R. China. ${ }^{2}$ School of Electrical Engineering, Hebei University of Science and Technology, Shijiazhuang, Hebei 050018, P.R. China.

\section{Acknowledgements}

The authors express their sincere thanks to the referees for the careful and detailed reading of the manuscript and very helpful suggestions. The project was supported by the Natural Science Foundation of China (11371120), the Natural Science Foundation of Hebei Province (A2013208147, F2014208042, A2015208051) and the Education Department of Hebei Province Science and Technology Research Project (Z2014095, Z2014060).

Received: 31 July 2014 Accepted: 19 December 2014 Published online: 30 January 2015

\section{References}

1. Gafiychuk, V, Datsko, B, Meleshko, V: Mathematical modeling of time fractional reaction-diffusion systems. J. Comput. Appl. Math. 220, 215-225 (2008)

2. Gejji, VD: Positive solutions of a system of non-autonomous fractional differential equations. J. Math. Anal. Appl. 302, 56-64 (2005)

3. Ibrahim, RW, Darus, M: Subordination and superordination for univalent solutions for fractional differential equations J. Math. Anal. Appl. 345, 871-879 (2008)

4. Ladaci, S, Loiseau, JL, Charef, A: Fractional order adaptive high-gain controllers for a class of linear systems. Commun. Nonlinear Sci. Numer. Simul. 13, 707-714 (2008)

5. Lazarević, MP: Finite time stability analysis of $\mathrm{PD}^{\alpha}$ fractional control of robotic time-delay systems. Mech. Res. Commun. 33, 269-279 (2006)

6. Podlubny, I: Fractional Differential Equations. Academic Press, San Diego (1999)

7. Rida, SZ, El-Sherbiny, HM, Arafa, AAM: On the solution of the fractional nonlinear Schrödinger equation. Phys. Lett. A 372, 553-558 (2008)

8. Samko, SG, Kilbas, AA, Marichev, Ol: Fractional Integrals and Derivatives: Theory and Applications. Gordon \& Breach, Yverdon (1993)

9. Benchohra, M, Slimani, BA: Existence and uniqueness of solutions to impulsive fractional differential equations. Electron. J. Differ. Equ. 2009, 10 (2009)

10. Nakhushev, MA: The Sturm-Liouville problem for a second order ordinary differential equation with fractional derivatives in the lower terms. Dokl. Akad. Nauk SSSR 234, 308-311 (1977)

11. Aleroev, TS: The Sturm-Liouville problem for a second order ordinary differential equation with fractional derivatives in the lower terms. Differ. Uravn. 18, 341-342 (1982)

12. Su, XW: Boundary value problem for a coupled system of nonlinear fractional differential equations. Appl. Math. Lett. 22, 64-69 (2009)

13. Zhang, SQ: Existence of solution for a boundary value problem of fractional order. Acta Math. Sci. Ser. B 26, 220-228 (2006)

14. Zhang, SQ: Positive solutions for boundary-value problems of nonlinear fractional differential equations. Electron. J. Differ. Equ. 2006, 36 (2006)

15. Yang, L, Shen, CF, Xie, DP: Multiple positive solutions for nonlinear boundary value problem of fractional order differential equation with the Riemann-Liouville derivative. Adv. Differ. Equ. 2014, 284 (2014)

16. Zhao, KH, Gong, P: Positive solutions of Riemann-Stieltjes integral boundary problems for the nonlinear coupling system involving fractional-order differential. Adv. Differ. Equ. 2014, 254 (2014)

17. Wang, JH, Xiang, HJ, Chen, FL: Existence of positive solutions for a discrete fractional boundary value problem. Adv. Differ. Equ. 2014, 253 (2014)

18. Bai, ZB, Lü, HS: Positive solutions for boundary value problem of nonlinear fractional differential equation. J. Math. Anal. Appl. 311, 495-505 (2005)

19. Li, CF, Luo, XN, Zhou, Y: Existence of positive solutions of the boundary value problem for nonlinear fractional differential equations. Comput. Math. Appl. 59, 1363-1375 (2010)

20. Leggett, RW, Williams, LR: Multiple positive fixed points of nonlinear operators on ordered Banach spaces. Indiana Univ. Math. J. 28, 673-688 (1979)

21. Podlubny, I: Fractional Differential Equations. Mathematics in Science and Engineering. Academic Press, New York (1999)

22. Kilbas, AA, Srivastava, HM, Trujillo, JJ: Theory and Applications of Fractional Differential Equations. North-Holland Mathematics Studies. Elsevier, Amsterdam (2006) 